\title{
The National COPD Audit - what you need to know 䇺
}

\author{
Authors: John R Hurst, ${ }^{A}$ Viktoria McMillan ${ }^{B}$ and $C$ Michael Roberts ${ }^{C}$
}

The secondary care work stream of the National COPD Audit Programme aims to improve care and outcomes for patients with exacerbation of chronic obstructive pulmonary disease (COPD) wherever and whenever they are admitted to hospital. To achieve this, prospective audit is combined with real-time feedback of data to individual units, together with support for quality improvement and high-level change levers. COPD exacerbations comprise a large proportion of the acute take. Only by working collaboratively across emergency, acute and general medicine, respiratory, geriatric and other teams can individual trusts deliver optimal care. This review provides background to the national COPD audit programme, relevant to all those caring for people with COPD exacerbations in secondary care.

KEYWORDS: COPD, quality improvement, NACAP

\section{Introduction}

Chronic obstructive pulmonary disease (COPD) causes a huge burden of morbidity and mortality in the UK, as it does globally. COPD is the end result of a person with (complex) genetic susceptibility being exposed to sufficient environmental stimulus which, in high-income countries, is usually tobacco smoke. COPD is part of a spectrum of smoking-related lung diseases that encompasses chronic bronchitis and emphysema, and COPD is diagnosed when (and only when) there is persistent airflow obstruction on post-bronchodilator spirometry in the context of an appropriate clinical syndrome. ${ }^{1}$

Much of the morbidity and mortality in COPD relates to episodes of deterioration in respiratory health, termed exacerbations. Exacerbation is a clinical diagnosis of exclusion made where other causes of symptom changes have been considered and, where appropriate, excluded.

Exacerbations are one of the commonest reasons for emergency hospital admission in the UK. NHS England data suggest COPD is responsible for 115,000 emergency admissions per year, over 1 million bed days and 16,000 deaths within 90 days of admission. ${ }^{2}$

Authors: A professor of respiratory medicine, University College London, London, UK; ${ }^{\text {B }}$ rogramme manager, Royal College of Physicians, London, UK; ${ }^{C}$ consultant integrated respiratory physician, The Princess Alexandra Hospital NHS Trust, Harlow, UK and NACAP senior clinical lead, Royal College of Physicians, London, UK
As in other areas of respiratory medicine, the UK has had a long standing audit programme with 'snap shot' COPD exacerbation audits coordinated by the Royal College of Physicians (RCP) running since 1997 and completed again in 2003, 2008 and 2014. While these documented incremental improvements in key clinical outcomes, the inability of this scheme to support transformational quality improvement in this highest burden area of respiratory practice was a key driver to the introduction of a rolling national audit from February 2017, tied to a best-practice tariff (financial incentives) for trusts not meeting specified performance targets. The scope of the audit programme was subsequently extended from secondary care COPD to include primary care COPD in Wales, pulmonary rehabilitation and, more recently, asthma - in adults, and in children and young people. Together, these audits form the National Asthma and COPD Audit Programme (NACAP).

\section{The COPD audit in context}

There are a variety of NHS initiatives designed to improve quality of care and it is important to understand where NACAP fits within overarching strategy. NACAP is part of the National Clinical Audit and Patient Outcomes Programme (NCAPOP). NCAPOP is a portfolio of over 30 national audits, funded by NHS England and the Welsh government, and (for some) the devolved governments of Scotland and Northern Ireland. NACAP includes England, Scotland and Wales but not Northern Ireland, and is commissioned through the Healthcare Quality Improvement Partnership (HQIP). The funding for NCAPOP is top-sliced from trust budgets, participation in NCAPOP audits is mandated, and the results form part of trust board quality accounts and as such should be included within their annual report. The rationale behind topic selection for NCAPOP includes areas where there is evidence of variable and/or poor performance and where improvement would provide significant benefits to patients and to the NHS. COPD has been part of the programme since 2013 and asthma was added in 2018. The RCP delivers both the 2013 COPD audit and the 2018 combined asthma and COPD audit programmes, NACAP.

NCAPOP is just one strand in a series of assurance and improvement processes within the NHS requiring data collection that can add to both the burden and confusion of purpose felt on the front line. The NACAP team has worked to try and align audit data with other data collection requirements and to secure incentives that support audit and quality improvement linked to COPD and asthma care. For example, NACAP has agreed a national COPD data set with the Care Quality Commission to be applied to secondary care provider inspections. Units that 
participate in the audit and demonstrate good quality care are more likely to have a light touch approach to their inspections. Discussions are ongoing with the Getting It Right First Time team to avoid duplicate data collection and to use NACAP data to support trust visits and quality improvement recommendations made as a result. In Wales, audit data are used to support the Respiratory Health Implementation Group to set national quality improvement aims. Similar engagement is underway with Scottish respiratory networks.

This focus on quality improvement is a key change in the approach to national audit exemplified by NACAP and is a primary reason why all physicians involved in the management of asthma or COPD patients should feel challenged to engage in the programme. The NACAP improvement strategy has four intervention streams.

- Data to support improvement.

- Quality improvement methodological support to front-line teams.

- Use of high-level change levers.

- Engaging with patients and the public.

Traditionally, national audit has been perceived by clinicians as a data collection exercise to produce reports for national use with often limited local value. Timelines were lengthy, with report publication dates as long as a full year after data were collected. Audit rounds were cross-sectional with intervals of 1-5 years between data collection periods. As a result, data were often perceived as out of date and less relevant to current practice. Interventions made on the basis of baseline data might have an unknown impact for years before a further data collection and reporting round provided comparisons. There is now a strong evidence base that tells us this form of clinical audit has limited impact on improving the quality of care patients receive and on improving their outcomes. ${ }^{3}$

NACAP is different and one of a new breed of audits that recognise that the purpose of data collection is to support quality improvement, not only at a national level, but at regional and, most importantly, local site level too. To deliver this, the audit has undergone a total revolution in approach. Gone are the periodic cross-sectional data collection periods in favour of continuous data collection. This allows participants to feed data into the system in real time and, in return, to see their data online within a few minutes of data entry. Data are reported back in the form of run charts, updated every 15 minutes, to provide data in a format that supports quality improvement. Run charts map performance against a quality indicator on the y axis against time lines presented on the $x$ axis (Fig 1). This allows participating teams to not only assess the quality of their service in real time but also compare against past performance and to benchmark against others. ${ }^{4}$ If a change is made to improve services the effect can be seen in real time rather than waiting for months or years to determine the impact. Data lines are also shown for overall national results that can be broken down to England, Scotland or Wales, and data for an aspirational target are also provided where relevant.

Bespoke annual reports are provided for each participating trust. Overall reports and, in England, reports for each Sustainability and Transformation Partnership are also produced. ${ }^{5}$ In Scotland and Wales, local health board reports designed to inform commissioners of the care standard in their geography support them to contract for improvement. Such reports are short and highlight key findings using infographics and define a small number of clearly indicated national improvement aims.

The continuous nature of the data entry, although more burdensome than a cross-sectional audit, provides sufficient case numbers for each unit to have confidence in the relevance of their own data. This approach empowers local teams to deliver more effective care and quality improved services. ${ }^{6,7}$ The trade-off for continuous collection has been a radical reduction in the number and complexity of the data collected in order to limit the burden. Each measure has been selected by the clinical community and discussed with a patient panel. This was on the basis of previous audits demonstrating unwarranted variability and suboptimal performance for an intervention that, if made, has a strong evidence base for improving quality of care and/or outcomes. The smaller number of indicators collected also provides a more focused and reasonable improvement target for teams to achieve rather than the past audit reporting on multiple areas for improvement.

Data collection and real time presentation in a format that is meaningful and relevant are critical elements of any improvement process but NACAP goes beyond this to provide quality improvement tools to support teams in their efforts to improve care to their patients. NACAP has delivered a series of regional quality improvement workshops in 2018 and is in process
Fig 1. Example chronic obstructive pulmonary disease audit run chart. The dark blue line is the proportion of patients receiving specialist review, and the mid-green line is the proportion receiving specialist review within 24 hours. The mid-blue line is the proportion of patients receiving a discharge bundle. Provision of specialist review within 24 hours and a discharge bundle constitutes best practice tariff. The red line indicates the $60 \%$ best practice tariff target, and actual performance is indicated by the area below the dark green line. $\mathrm{BPT}=$ best practice tariff.

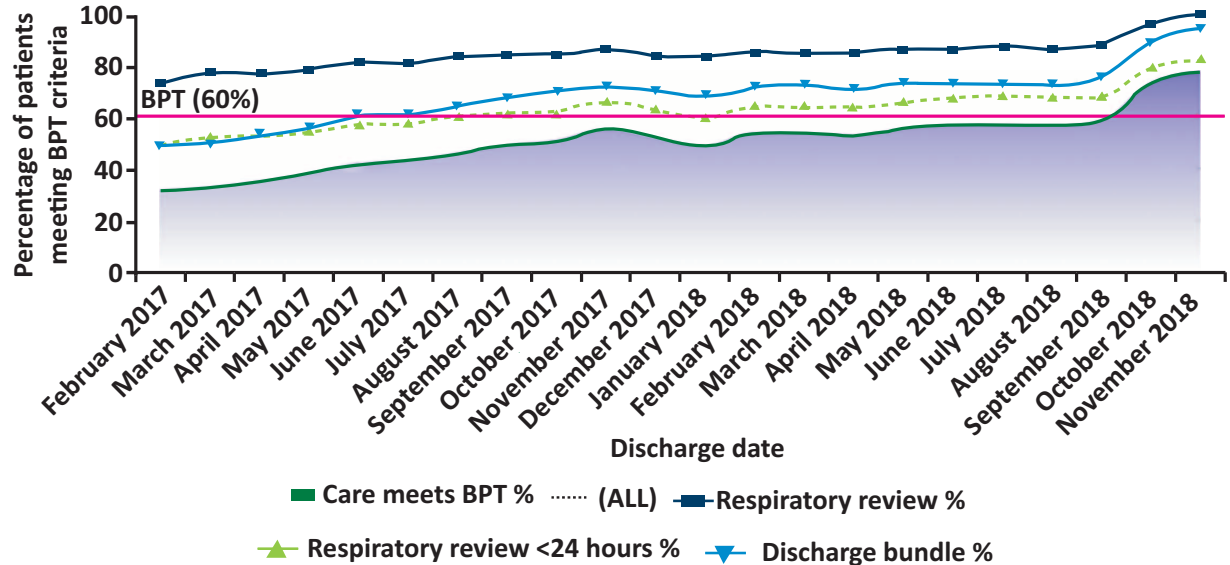


of delivering more this year and next. Teams of clinicians and managers are invited to learn about an approach for improvement using 'plan, do, study, act' (model for improvement) methodology and at the end of the day return to their units with a small test of change improvement plan. ${ }^{8}$ To further support clinicians in their quest for improvement, NACAP has a good practice repository with case histories and contact details for teams who have achieved excellence in various key measures. The NACAP team have also contributed a number of teaching elements to the nationally commissioned Welsh quality improvement web tool that is used to spearhead the national Welsh quality improvement campaign in primary care.

The use of high-level change levers is exemplified by a number of NACAP engagement initiatives. In order to promote audit participation and improvement, a best practice tariff (BPT) was negotiated with NHS England for COPD hospital care. The BPT provides a high-level change lever. To further support quality improvement, the team has presented the audit data to National Institute for Health and Care Excellence who have used the evidence to set quality standards for COPD care, and to the Quality and Outcomes Framework review panel to improve primary care incentives. NACAP has also supported a pulmonary rehabilitation accreditation programme that launches in 2019, while NHS Improvement has mandated a COPD discharge bundle initiative to be delivered through the Academic Health Science Network.

The fourth strand of the NACAP improvement programme is to better engage with patients and the public to drive improvement in asthma and COPD services. NACAP works closely with Asthma UK, The British Lung Foundation, the Royal College of Paediatrics and Child Health and the Royal College of General Practitioners. Our patient panel has defined the areas patients and carers see as priorities for service improvement in COPD; adult, children and young peoples' asthma. These are then added into our datasets, reporting and improvement aims.

\section{Selection of audit variables}

As described above, the audit dataset is a compromise between collecting a wider range of data fields, and the burden data collection places on hard-working, front-line clinical teams. As such, the strategy was to minimise fields and to include fields that tracked most closely with outcomes. The intention ultimately is to link audit data to other datasets to allow additional fields to be pulled from, for example, the primary care record. A second principle has been that the audit dataset would be periodically revised, removing fields that were not adding value (for example by being poorly completed or universally met), and to reflect changing quality improvement priorities. This has occurred once since the launch of the rolling COPD audit and the revised dataset explicitly included questions on the presence and management of key cardiac and mental health comorbidity while, for example, questions on acute medicine review were removed as patients were almost universally seen by a member of the acute medicine team.

The current secondary care COPD dataset is listed in Table 1.

The BPT considered only a specialist review within the first 24 hours (COPD specialist being locally defined, and multiprofessional) and a discharge bundle prior to discharge. The latter should include smoking cessation support, inhaler technique check, referral to pulmonary rehabilitation and follow-up arrangements. The initial target was for this to be delivered in $60 \%$ or more of
Table 1. The current secondary care chronic

obstructive pulmonary disease audit dataset

$\begin{array}{ll}\text { Arrival information } & \text { Date and time of arrival at hospital } \\ \text { Patient data } & \text { NHS number, date of birth, gender, } \\ & \text { home postcode, smoking status }\end{array}$

NEWS2 score on admission

\begin{tabular}{|c|c|}
\hline $\begin{array}{l}\text { Admission } \\
\text { information }\end{array}$ & Date and time of admission to unit \\
\hline $\begin{array}{l}\text { Respiratory } \\
\text { specialist review }\end{array}$ & Date and time of first specialist review \\
\hline Oxygen & $\begin{array}{l}\text { Presence of oxygen prescription, target } \\
\text { saturation range and use of oxygen }\end{array}$ \\
\hline $\begin{array}{l}\text { Non-invasive } \\
\text { ventilation }\end{array}$ & Date and time of NIV if appropriate \\
\hline Spirometry & $\begin{array}{l}\text { Most recent } F E V_{1} \text { per cent predicted, } \\
\text { and } F E V_{1} / F V C \text { ratio with date }\end{array}$ \\
\hline Comorbidity & $\begin{array}{l}\text { Presence of and new intervention for } \\
\text { cardiovascular disease and mental illness }\end{array}$ \\
\hline Discharge & $\begin{array}{l}\text { Date of discharge (or death), completion } \\
\text { of a discharge bundle including specific } \\
\text { examples of good practice }\end{array}$ \\
\hline
\end{tabular}

$\mathrm{FEV}_{1}=$ forced expiratory volume in 1 second; $\mathrm{FVC}=$ forced vital capacity; NEWS2 = National Early Warning Score 2; NIV = non-invasive ventilation

the audited cases. Case ascertainment is dealt with separately and compares the number of coded admissions with the number of audit admissions. The best performing trusts are those with good BPT performance and high case ascertainment. Low case ascertainment can reflect a number of challenges, including inaccurate coding.

The rolling clinical audit is complemented by a cross-sectional organisational dataset. Where questions are common to COPD and asthma audits, they have been asked in the same way and the organisational audit is a single dataset for both conditions.

It is critical to point out that the purpose of the audit is to drive local quality improvement. Therefore, the intention is that audit data are collected in real time during the admission and entered on to the portal shortly after discharge. This provides real-time performance data, benchmarked against national performance, and provides a tool by which to monitor quality improvement initiatives.

\section{Key messages for the acute and general physician}

Given the high number of COPD admissions, and readmissions, exacerbations constitute a high proportion of the acute take and managing COPD effectively is a key skill for acute and general physicians; optimal delivery of COPD care is not just the responsibility of specialist respiratory teams. Certain aspects of the audit, notably timely application of non-invasive ventilation (NIV) and appropriate oxygen prescription, and identification of patients for early specialist review fall directly within the first 24 hours and therefore should be considered markers of acute medicine quality. Non-respiratory physicians, and the teams they work in, responsible for any elements of COPD and asthma care should be just as engaged in a quality improvement programme as respiratory colleagues. Each trust should consider how best to 
use the audit to drive quality improvement for people admitted with COPD exacerbations, wherever they are admitted and under whichever team, be that acute medicine, geriatrics, respiratory medicine or others. This is particularly relevant to evening and weekend working, in which some people with exacerbations may be discharged quickly and before they have had specialist review.

There are three current quality improvement priorities for the secondary care COPD audit. The first is to challenge teams to access previous spirometry to confirm that a patient being managed for an exacerbation has a robust diagnosis. This information is currently unavailable in $60 \%$ of admissions and even where this is available, COPD is not confirmed in $12 \%$. The second challenge is timely provision of NIV in those admitted with decompensated, acidotic hypercapnic respiratory failure. Late NIV is associated with poor outcomes and the current target of 2 hours or less is currently being met in only $21 \%$ of cases (with the caveat that these include late deteriorations). The third quality improvement priority is to ensure all smokers are offered smoking cessation pharmacotherapy, and this is currently provided in just $67 \%$ of smokers and accepted in just $25 \%$.

Twenty-five per cent of people discharged are readmitted within 30 days and key factors associated with this include the presence of comorbidities, previous hospitalisation and length of stay (higher risk with longer length of stay). While not in the top three quality improvement priorities, the emphasis of the audit on the discharge bundle and assessment and management of comorbidity are designed to reduce the risk of readmissions.

To further emphasise the key role of non-respiratory physicians, the soon to be introduced first hour of care standards, that will largely replace the 4-hour emergency department (ED) standard, will be assessing the care given by physicians based in ED and acute medical units who manage asthma admissions regardless of specialty training! We strongly recommend discussion between ED, acute and general medicine and respiratory colleagues about how to work together to give the best possible care to patients with asthma and COPD.

The NACAP run charts are available to all by requesting a login from the site lead and we suggest exploring your own data in detail and sharing results with the teams with which you work. Consider the implications for areas of care requiring improvement. Discuss with colleagues a plan for improvement. Implement change and monitor the impact using real time feedback from the run charts. NACAP provides the data to help identify the issues, the tools and best practice examples for you to consider and adopt locally and run charts to demonstrate if the changes you have made have made a difference. NACAP quality improvement workshops are free and we welcome all who want to make a difference.

Ultimately NACAP is about patient care. People admitted to hospital with admission of COPD deserve excellent care this time and every time, wherever and whenever they are admitted.

\section{References}

1 Global Initiative for Chronic Obstructive Lung Disease, 2019. https://goldcopd.org [Accessed 19 June 2019].

2 NHS England. Overview of potential to reduce lives lost from chronic obstructive pulmonary disease (COPD). NHS, 2014. www.england.nhs.uk/wp-content/uploads/2014/02/rm-fs-6.pdf [Accessed 19 June 2019].

3 Ivers N, Jamvedt G, Flottorp S et al. Audit and feedback: effects on professional practice and healthcare outcomes. Cochrane Database Syst Rev 2012:CD000259.

4 Gude WT, Brown B, van der Veer SN et al. Clinical performance comparators in audit and feedback: a review of theory and evidence. Implement Sci 2019;14:39.

5 Royal College of Physicians. National Asthma and COPD Audit Programme (NACAP) resources. London: RCP, 2018. www. rcplondon.ac.uk/projects/outputs/national-asthma-and-copd-auditprogramme-nacap-resources [Accessed 19 June 2019].

6 Brehaut JC, Colquhoun HL, Eva KW et al. Practice feedback interventions: 15 suggestions for optimizing effectiveness. Ann Intern Med 2016;164:435-41.

7 National Institute for Health and Clinical Excellence. How to change practice. NICE, 2007.

8 Institute for Healthcare Improvement. How to improve: science of improvement: testing changes. IHI. www.ihi.org/resources/Pages/ HowtoImprove/ScienceofImprovementTestingChanges.aspx [Accessed 19 June 2019].

Address for correspondence: Prof John R Hurst, UCL Respiratory, Royal Free Campus, London NW3 2PF, UK. Email:j.hurst@ucl.ac.uk 